\title{
A Fourier restriction estimate for surfaces of positive curvature in $\mathbb{R}^{6}$
}

\author{
Faruk Temur
}

\begin{abstract}
We improve the best known exponent for the restriction conjecture in $\mathbb{R}^{6}$, improving the recent results of Bourgain and Guth. The proof is applicable to any dimension $n$ satisfying $n \equiv 0 \bmod 3$.
\end{abstract}

\section{Introduction}

In [7] Stein posed the following well-known conjecture. Let $S \subset \mathbb{R}^{n}$ be a smooth, compact hypersurface with positive definite second fundamental form and let $\sigma$ be its surface measure. Then, for $p>2 n / n-1$ and $f \in L^{\infty}(S, \sigma)$, we have

$$
\|\widehat{f d \sigma}\|_{p} \leq C_{p, S}\|f\|_{\infty}
$$

This conjecture is related to some other important problems in harmonic analysis and PDE such as the Kakeya conjecture, the Bochner-Riesz conjecture, and the local smoothing problem; see [3], [6], [9], [14]. For $n=2$ it is known to be true; see [5]. For $n \geq 3$ it is open despite much effort. The first progress towards this case was the Tomas-Stein theorem, and gives $p>2(n+1) /(n-1)$; see [12]. In [2] Bourgain was able to go below this exponent. Wolff improved Bourgain's result to $\left(2 n^{2}+n+6\right) /\left(n^{2}+n-1\right)$; see [13]. Then in three dimensions Tao, Vargas, and Vega further lowered this exponent, and more importantly they developed the bilinear approach which related this conjecture to restriction estimates for compact, transverse subsets of hypersurfaces; see [10], [11]. The work of Tao in [8], which was a bilinear estimate for compact transverse subsets of paraboloids, through this bilinear method, verified the conjecture for $p>2(n+2) / n$. This exponent is the best one can obtain from that approach.

In [1], Bennett, Carbery, and Tao posed a multilinear version of the restriction conjecture and solved it. Let $S_{1}, \ldots, S_{m} \subset \mathbb{R}^{n}$ be smooth compact hypersurfaces that are transverse, that is for any choice of points $\left\{x_{i} \in S_{i}\right\}$ we have $\mid x_{1}^{\prime} \wedge \cdots \wedge$ $x_{m}^{\prime} \mid>c$ where $x_{i}^{\prime}$ is the unit normal at $x_{i}$, and $c$ is some positive constant. Let $\sigma_{i}$ 
be the surface measure of $S_{i}$. Then for $q>2 m / m-1$ and $p^{\prime} \leq q(m-1) / m$ the result of [1] implies

$$
\left\|\prod_{i=1}^{m} \widehat{f_{i} d \sigma_{i}}\right\|_{L^{q / m}\left(\mathbb{R}^{n}\right)} \lesssim \prod_{i=1}^{m}\left\|f_{i}\right\|_{L^{p}\left(S_{i}\right)} .
$$

This result by itself does not imply any progress towards the restriction conjecture, but recently, in [4], Bourgain and Guth combining this with the idea of rescaling, significantly improved the known exponents for $n>4$. The exponents given in [4] are for every $n>2$ as follows:

$$
\begin{aligned}
& p>\frac{8 n+6}{4 n-3} \text { if } n \equiv 0 \quad \bmod 3, \\
& p>\frac{2 n+1}{n-1} \text { if } n \equiv 1 \quad \bmod 3, \\
& p>\frac{4 n+4}{2 n-1} \quad \text { if } n \equiv 2 \quad \bmod 3 .
\end{aligned}
$$

For $n=3,4$ this does not give any improvement, but refining their analysis and combining it with Wolff's Kakeya maximal function estimate in [13], Bourgain and Guth, for $n=3$, improved the known exponent $p>10 / 3$ to $p>33 / 10$. The aim of this paper is to show that this refined method can be used for $n=6$ too, and to calculate the improvement. We shall also make it clear how to use this strategy for any dimension $n \equiv 0 \bmod 3$, though as the improvement is very small and the process is very technical we will not calculate the improvement for general $n$. We state the case in $n=6$ as a theorem:

Theorem 1. Let $S \subset \mathbb{R}^{6}$ be a smooth, compact hypersurface with positive definite second fundamental form and $\sigma$ its surface measure. Then for $p>18 / 7-2 / 735$ and $f \in L^{\infty}(S, \sigma)$ we have

$$
\|\widehat{f d \sigma}\|_{p} \lesssim\|f\|_{\infty}
$$

Thus we have improved the Bourgain-Guth exponent by $2 / 735$.

The rest of the paper is organized as follows. In Section 2, we describe the proof by Bourgain and Guth of (1.3), and point out what allows us when $n \equiv 0 \bmod 3$ to improve this. In Section 3 we calculate explicitly the improvement for $n=6$, and at the end of that section, it will be clear to the reader that the process can be repeated to obtain improvement for any $n$ with $n \equiv 0 \bmod 3$.

\section{The Bourgain-Guth argument}

We first remark that standard $\epsilon$-removal arguments allow us to derive Theorem 1 from the following theorem. 
Theorem 2. Let $S \subset \mathbb{R}^{6}$ be a smooth, compact hypersurface with positive definite second fundamental form and let $\sigma$ be its surface measure. Then, for $p \geq 18 / 7-$ $2 / 735$, and $f \in L^{\infty}(S, \sigma)$ we have

$$
\|\widehat{f d \sigma}\|_{L^{p}(B(0, R))} \lesssim R^{\epsilon}\|f\|_{\infty} .
$$

While working in this localized setting we shall use the following version of (1.2) proved in [1]: for $S_{1}, S_{2}, \ldots, S_{m}$ satisfying the same properties as described in section 1 , one has, for every $\epsilon>0$,

$$
\left\|\prod_{i=1}^{m} \widehat{f_{i} d \sigma_{i}}\right\|_{L^{q / m}(B(0, R))} \lesssim R^{\epsilon} \prod_{i=1}^{m}\left\|f_{i}\right\|_{L^{2}\left(S_{i}\right)}
$$

for $q \geq 2 m / m-1$.

We further remark that it suffices to prove Theorem 2 for $\|f\|_{\infty} \leq 1$, and we accordingly define $Q_{R}^{p}$ to be the best constant satisfying

$$
\|\widehat{f d \sigma}\|_{L^{p}(B(0, R))} \leq Q_{R}^{p}
$$

This constant clearly is well defined by the crude estimate

$$
\|\widehat{f d \sigma}\|_{L^{p}(B(0, R))} \lesssim R^{n / p} .
$$

Thus we reduce to showing that $Q_{R}^{p} \lesssim R^{\epsilon}$.

We continue with several lemmas, proofs of which can be found in [4]. The first two lemmas rely on multilinear estimates from [1], while the third uses rescaling.

Let $S$ be a compact, smooth hypersurface in $\mathbb{R}^{n}$ with positive definite second fundamental form. For $x \in S$ let $x^{\prime} \in S^{n-1}$ denote the unit normal to the surface at the point $x$, and let $\Gamma: S^{n-1} \rightarrow S$ be the Gauss map. Hence $\Gamma\left(x^{\prime}\right)=x$. In what follows we will use the notation $\oint_{E}$ to denote the average over the set $E$. Now we are ready to state our first lemma.

Lemma 1. Let $U_{i} \subset S, 1 \leq i \leq n$, be small caps such that $\left|x_{1}^{\prime} \wedge \cdots \wedge x_{n}^{\prime}\right|>c$ for all $x_{i} \in U_{i}$. Let $D_{i} \subset U_{i}, 1 \leq i \leq n$, be discrete sets of $1 / M$-separated points for $M$ large. Then, for a bounded function a on $S$,

$$
\oint_{B_{M}} \prod_{i=1}^{n}\left|\sum_{\xi \in D_{i}} a(\xi) e^{-i x \cdot \xi}\right|^{2 /(n-1)} \ll M^{\epsilon} \prod_{i=1}^{n}\left(\sum_{\xi \in D_{i}}|a(\xi)|^{2}\right)^{1 /(n-1)},
$$

where $B_{M} \subset \mathbb{R}^{n}$ is a ball of radius $M$.

This lemma is a discretized version of (1.2), using the uncertainty principle one replaces discrete sums with integrals of functions that are constant on $1 / M$ neighborhoods of points $\xi$, and then applies (1.2). For details see [4].

Lemma 2. Let $2 \leq m \leq n$ and let $V$ be an $m$-dimensional subspace of $\mathbb{R}^{n}$. Let $P_{1} \ldots P_{m} \in S$ be points such that $P_{i}^{\prime} \in V$, for all $1 \leq i \leq m$, and $\left|P_{1}^{\prime} \wedge \cdots \wedge P_{m}^{\prime}\right|>c$. 
Let $U_{1}, \ldots, U_{m} \subset S$ be small neighborhoods of $P_{1}, \ldots, P_{m}$. Let $M$ be large and let $D_{i} \subset U_{i}$ be sets of $1 / M$ separated points $\xi$ that satisfy the condition $\operatorname{dist}\left(\xi^{\prime}, V\right)$ $<c / M$. Then for $f_{i} \in L^{\infty}\left(U_{i}\right)$, we have

$$
\begin{aligned}
\oint_{B_{M}} & \prod_{i=1}^{m}\left|\sum_{\xi \in D_{i}} \int_{|\zeta-\xi|<c / M} f_{i}(\zeta) e^{-i x \cdot \zeta} d \sigma(\zeta)\right|^{2 /(m-1)} d x \\
& \ll M^{\epsilon}\left(\oint_{B_{M}} \prod_{i=1}^{m}\left(\sum_{\xi \in D_{i}}\left|\int_{|\zeta-\xi|<c / M} f_{i}(\zeta) e^{-i x \cdot \zeta} d \sigma(\zeta)\right|^{2}\right)^{1 /(2 m)} d x\right)^{2 m /(m-1)}
\end{aligned}
$$

To prove this lemma we first use the uncertainty principle to discretize it as in Lemma 1 , then by the hypothesis $\operatorname{dist}\left(\xi^{\prime}, V\right)<c / M$ and the uncertainty principle make a dimensional reduction to $\mathbb{R}^{m}$, and finally apply Lemma 1 in $\mathbb{R}^{m}$. Again for details we refer to [4]. Finally we state the following lemma, which follows from parabolic rescaling.

Lemma 3. Let $U_{\rho}$ be a cap of radius $\rho$ on $S$. Then

$$
\left\|\int_{U_{\rho}} f(\xi) e^{-i x \cdot \xi} d \sigma(\xi)\right\|_{L^{p}\left(B_{R}\right)} \leq \rho^{n-1-(n+1) / p} Q_{\rho R}^{p}
$$

Now we are in a position to describe the Bourgain-Guth argument. Let $f \in$ $L^{\infty}(S)$ satisfy $|f| \leq 1$ and let $x \in B_{R}$. Let

$$
R^{\epsilon} \gg K_{n} \gg K_{n-1} \gg \cdots \gg K_{1}
$$

be constants independent of $f$ that will be specified later. Decompose $S$ into caps $U_{\alpha}^{n}$ of size $1 / K_{n}$, and let $\xi_{\alpha}^{n} \in U_{\alpha}^{n}$. Thus

$$
\int_{S} f(\xi) e^{-i x \cdot \xi} \sigma(d \xi)=\sum_{\alpha} e^{-i x \cdot \xi_{\alpha}^{n}} \int_{U_{\alpha}^{n}} f(\xi) e^{-i x \cdot\left(\xi-\xi_{\alpha}^{n}\right)} d \sigma(\xi)=: \sum_{\alpha} e^{-i x \cdot \xi_{\alpha}^{n}} T_{\alpha}^{n} f(x) .
$$

Take a function $\eta \in \mathcal{S}\left(\mathbb{R}^{n}\right)$ with $\widehat{\eta}(x)=1$ on $B(0,1)$ and $\widehat{\eta}(x)=0$ outside $B(0,2)$. Let $\eta_{r}(x)=\frac{1}{r^{n}} \eta(x / r)$ so that

$$
T_{\alpha}^{n} f(x)=T_{\alpha}^{n} f * \eta_{K_{n}} .
$$

For fixed $x$, using the Bernstein inequality we can write

$$
\begin{aligned}
\left|T_{\alpha}^{n} f(x)\right| & \leq \int\left|T_{\alpha}^{n} f(x-y) \eta_{K_{n}}(y)\right| d y \\
& \lesssim \int\left|T_{\alpha}^{n} f(x-y) \eta_{K_{n}}(y)\right|^{1 / n}\left|T_{\alpha}^{n} f(x-y) \eta_{K_{n}}(y)\right|^{(n-1) / n} d y \\
& \leq\left(\int\left|T_{\alpha}^{n} f(x-y) \eta_{K_{n}}(y)\right|^{1 / n} d y\right)\left\|T_{\alpha}^{n} f(x-\cdot) \eta_{K_{n}}(\cdot)\right\|_{\infty}^{(n-1) / n} \\
& \lesssim\left(\int\left|T_{\alpha}^{n} f(x-y) \eta_{K_{n}}(y)\right|^{1 / n} d y\right)\left\|T_{\alpha}^{n} f(x-\cdot) \eta_{K_{n}}(\cdot)\right\|_{1}^{(n-1) / n} K_{n}^{1-n} .
\end{aligned}
$$


Thus if $\left|T_{\alpha}^{n} f(x)\right|$ is nonzero we can use division to write

$$
\left(\int\left|T_{\alpha}^{n} f(x-y) \eta_{K_{n}}(y)\right| d y\right)^{1 / n} \lesssim\left(\int\left|T_{\alpha}^{n} f(x-y) \eta_{K_{n}}(y)\right|^{1 / n} d y\right) K_{n}^{1-n}
$$

Hence

$$
\left|T_{\alpha}^{n} f(x)\right| \lesssim\left(\int\left|T_{\alpha}^{n} f(x-y) \eta_{K_{n}}(y)\right|^{1 / n} d y\right)^{n} K_{n}^{n-n^{2}}
$$

This, of course, is trivial if $\left|T_{\alpha}^{n} f(x)\right|=0$, so it is independent of the value of $\left|T_{\alpha}^{n} f(x)\right|$. Taking the constant term inside the integral we obtain

$$
\lesssim\left(\int\left|T_{\alpha}^{n} f(x-y)\right|^{1 / n} \frac{1}{K_{n}^{n}}\left|\eta\left(\frac{y}{K_{n}}\right)\right|^{1 / n} d y\right)^{n}
$$

Define $\zeta(y)=\max _{\left|y-y^{\prime}\right| \leq 1}\left|\eta\left(y^{\prime}\right)\right|^{1 / n}$ and let $\zeta_{r}(y):=\frac{1}{r^{n}} \zeta(y / r)$. Then

$$
\left|T_{\alpha}^{n} f(x)\right| \lesssim\left(\int\left|T_{\alpha}^{n} f(x-y)\right|^{1 / n} \zeta_{K_{n}}(y) d y\right)^{n}=: c_{\alpha}^{n}(x)
$$

and

$$
\left|\int_{S} f(\xi) e^{i x \cdot \xi} d \sigma(\xi)\right| \lesssim \sum_{\alpha} c_{\alpha}^{n}(x) .
$$

We have $c_{\alpha}^{n}\left(x_{1}\right) \approx c_{\alpha}^{n}\left(x_{2}\right)$ whenever $\left|x_{1}-x_{2}\right|<K_{n}$.

For a fixed $x$ we have two possibilities:

1.i) There exist $\alpha_{1}, \ldots, \alpha_{n}$ such that $\left|\xi_{1}^{\prime} \wedge \cdots \wedge \xi_{n}^{\prime}\right|>c\left(K_{n}\right)$ for $\xi_{i} \in U_{\alpha_{i}}^{n}$ and

$$
c_{\alpha_{i}}^{n}(x)>K_{n}^{-n} \max _{\alpha} c_{\alpha}^{n}(x) .
$$

We can choose the same $\alpha_{1}, \ldots, \alpha_{n}$ for all $x$ in a ball of radius $K_{n}$ due to the fact that $c_{\alpha}^{n}(x)$ are constant on balls of this size.

1.ii) The negation of this, namely there exists an $(n-1)$-dimensional subspace $V_{n-1}$ such that

$$
c_{\alpha}^{n}(x) \leq K_{n}^{-n} \max _{\alpha} c_{\alpha}^{n}(x)
$$

if $\operatorname{dist}\left(U_{\alpha}^{n}, \widehat{V}_{n-1}\right) \gtrsim 1 / K_{n}$ where $\widehat{V}$ stands for the image of $V \cap S^{n-1}$ under the Gauss map. Since the $c_{\alpha}^{n}(x)$ are essentially constant on balls of radius $K_{n}$, on such balls we can take the linear subspace $V_{n-1}$ to be the same for all $x \in U_{\alpha}^{n}$.

If 1.i) holds, then since the number of caps is comparable to $K_{n}^{n-1}$,

$$
\left|\int_{S} f(\xi) e^{-i x \cdot \xi} \sigma(d \xi)\right| \lesssim K_{n}^{n-1} \max _{\alpha} c_{\alpha}^{n}(x) \lesssim K_{n}^{2 n-1}\left(\prod_{i=1}^{n} c_{\alpha_{i}}^{n}(x)\right)^{1 / n}
$$

and thus, letting $B_{1.1}$ denote $x \in B_{R}$ satisfying $\left.1 . \mathrm{i}\right)$,

$$
\int_{B_{1.1}}\left|\int_{S} f(\xi) e^{-i x \cdot \xi} \sigma(d \xi)\right|^{p} d x \lesssim K_{n}^{(2 n-1) p} \sum_{\alpha_{1}, \ldots, \alpha_{n}} \int_{B_{R}}\left(\prod_{i=1}^{n} c_{\alpha_{i}}^{n}(x)\right)^{p / n} d x
$$


Then by definition of $c_{\alpha_{i}}^{n}(x)$ and Hölder inequality we have

$$
\begin{aligned}
& \lesssim K_{n}^{(2 n-1) p} \sum_{\alpha_{1}, \ldots, \alpha_{n}} \int_{B_{R}}\left(\prod_{i=1}^{n} \int\left|T_{\alpha}^{n} f\left(x-y_{i}\right)\right|^{p / n} \zeta_{K_{n}}\left(y_{i}\right) d y_{i}\right) d x \\
& =K_{n}^{(2 n-1) p} \sum_{\alpha_{1}, \ldots, \alpha_{n}} \int_{B_{R}}\left(\int \prod_{i=1}^{n}\left|T_{\alpha}^{n} f\left(x-y_{i}\right)\right|^{p / n} \zeta_{K_{n}}\left(y_{i}\right) d y_{1} \ldots d y_{n}\right) d x
\end{aligned}
$$

By Fubini's theorem

$$
\lesssim K_{n}^{(2 n-1) p} \sum_{\alpha_{1}, \ldots, \alpha_{n}} \int\left(\int_{B_{R}} \prod_{i=1}^{n}\left|T_{\alpha}^{n} f\left(x-y_{i}\right)\right|^{p / n} d x\right) \prod_{i=1}^{n} \zeta_{K_{n}}\left(y_{i}\right) d y_{1} \ldots d y_{n} .
$$

Assuming $p \geq 2 n /(n-1)$, the inner integral of (2.2) satisfies

$$
\lesssim R^{\epsilon}
$$

Hence the main expression satisfies

$$
\lesssim K_{n}^{10 n^{2}} R^{\epsilon} \lesssim R^{2 \epsilon}
$$

The exponent $2 n / n-1$ is the one prescribed by the restriction conjecture, thus in this $n$-linear case we get the best possible exponent.

Now assume 1.ii) holds. Then, since the number of caps is comparable to $K_{n}^{n-1}$ we have

$$
\begin{aligned}
\left|\int_{S} f(\xi) e^{-i x \cdot \xi} d \sigma(\xi)\right| & \lesssim\left|\int_{\left\{\xi: \operatorname{dist}\left(\xi, \hat{V}_{n-1}\right) \lesssim 1 / K_{n}\right\}} f(\xi) e^{-i x \cdot \xi} d \sigma(\xi)\right|+\frac{1}{K_{n}} \max _{\alpha} c_{\alpha}^{n}(x) \\
& =\mathrm{I}+\mathrm{II} .
\end{aligned}
$$

Thus

$$
\begin{aligned}
\int_{B_{1.2}}\left|\int_{S} f(\xi) e^{-i x \cdot \xi} d \sigma(\xi)\right|^{p} d x \lesssim & \int_{B_{1.2}}\left|\int_{\left\{\xi: \operatorname{dist}\left(\xi, \hat{V}_{n-1}\right) \lesssim 1 / K_{n}\right\}} f(\xi) e^{-i x \cdot \xi} d \sigma(\xi)\right|^{p} d x \\
& +\frac{1}{K_{n}^{p}} \int_{B_{R}}\left(\max _{\alpha} c_{\alpha}^{n}(x)\right)^{p} d x
\end{aligned}
$$

We first evaluate the contribution coming from II. We have

$$
\frac{1}{K_{n}^{p}} \int_{B_{R}}\left(\max _{\alpha} c_{\alpha}^{n}(x)\right)^{p} d x \leq \frac{1}{K_{n}^{p}} \sum_{\alpha} \int_{B_{R}}\left(c_{\alpha}^{n}(x)\right)^{p} d x=\frac{1}{K_{n}^{p}} \sum_{\alpha}\left\|c_{\alpha}^{n}(x)\right\|_{L^{p}\left(B_{R}\right)}^{p},
$$

where the summation is over all caps of size $1 / K_{n}$. Using first the Hölder inequality and then Lemma 3 we have

$$
\begin{aligned}
\left\|c_{\alpha}^{n}(x)\right\|_{L^{p}\left(B_{R}\right)}^{p} & \lesssim \int_{B_{R}}\left(\int\left|T_{\alpha}^{n} f(x-y)\right|^{p} \zeta_{K_{n}}(y) d y\right) d x \\
& \lesssim \int\left(\int_{B_{R}}\left|T_{\alpha}^{n} f(x-y)\right|^{p} d x\right) \zeta_{K_{n}}(y) d y \lesssim K_{n}^{n+1-p(n-1)} Q_{R / K_{n}}^{p} .
\end{aligned}
$$


Hence the contribution of II is bounded by

$$
K_{n}^{n(2-p)} Q_{R / K_{n}}^{p}
$$

Since this is valid for all $f$, for an inductive argument aiming to bound $Q_{R}^{p}$ this is harmless when $p>2$.

To evaluate I we proceed as before, and decompose $S$ into caps $U_{\alpha}^{n-1}$ of radius $1 / K_{n-1}$ and let $\xi_{\alpha}^{n-1} \in U_{\alpha}^{n-1}$. Then

$$
\begin{aligned}
\int_{Y_{\hat{V}_{n-1}, K_{n}}} f(\xi) e^{-i x \cdot \xi} d \sigma(\xi) & =\sum_{\alpha} \int_{U_{\alpha}^{n-1} \cap Y_{\hat{V}_{n-1}, K_{n}}} f(\xi) e^{-i x \cdot \xi} d \sigma(\xi) \\
& =: \sum_{\alpha} e^{-i x \cdot \xi_{\alpha}^{n-1} \widetilde{T}_{\alpha}^{n-1} f(x)}
\end{aligned}
$$

where

$$
Y_{\hat{V}_{n-1}, K_{n}}:=\left\{\xi: \operatorname{dist}\left(\xi, \hat{V}_{n-1}\right) \lesssim 1 / K_{n}\right\} .
$$

On the other hand, let

$$
T_{\alpha}^{n-1} f(x)=\int_{U_{\alpha}^{n-1}} f(\xi) e^{-i x \cdot \xi} d \sigma(\xi) .
$$

Note a small difference between $\widetilde{T}$ and $T$. The first is defined on an intersection of caps with a strip, while the second on full caps. Defining $\eta_{K_{n-1}}$ and $\zeta_{K_{n-1}}$ in the same way as $\eta_{K_{n}}$ and $\zeta_{K_{n}}$, we write

$$
\begin{aligned}
\widetilde{c}_{\alpha}^{n-1}(x) & :=\left(\int\left|\widetilde{T}_{\alpha}^{n-1} f(x-y)\right|^{1 /(n-1)} \zeta_{K_{n-1}}(y) d y\right)^{n-1}, \\
c_{\alpha}^{n-1}(x) & :=\left(\int\left|T_{\alpha}^{n-1} f(x-y)\right|^{1 /(n-1)} \zeta_{K_{n-1}}(y) d y\right)^{n-1} .
\end{aligned}
$$

We shall need $c^{n-1}$ in the next step of our process. Via the same arguments as in in the definition of $c_{\alpha}^{n}$ we see that

$$
\left|\widetilde{T}_{\alpha}^{n-1} f(x)\right| \lesssim \widetilde{c}_{\alpha}^{n-1}(x), \quad \text { and } \quad\left|T_{\alpha}^{n-1} f(x)\right| \lesssim c_{\alpha}^{n-1}(x) .
$$

We again have two cases for a fixed $x$ :

2.i) There exist $\alpha_{1}, \ldots, \alpha_{n-1}$ such that $\left|\xi_{1}^{\prime} \wedge \cdots \wedge \xi_{n-1}^{\prime}\right|>c\left(K_{n-1}\right)$ for $\xi_{i} \in U_{\alpha_{i}}^{n-1}$, and

$$
\left|\widetilde{c}_{\alpha_{i}}^{n-1}(x)\right|>K_{n-1}^{-(n-1)} \max _{\alpha}\left|\widetilde{c}_{\alpha}^{n-1}(x)\right| .
$$

Since $c_{\alpha}^{n-1}$ are essentially constant on balls of size $K_{n-1}$ we can choose $\alpha_{1}, \ldots, \alpha_{n-1}$ the same for all $x$ in such balls.

2.ii) The negation of this, namely there exists an $(n-2)$-dimensional subspace $V_{n-2}$ which can be chosen to be a subspace of $V_{n-1}$ such that

$$
\left|\widetilde{c}_{\alpha}^{n-1}(x)\right| \leq K_{n-1}^{-(n-1)} \max _{\alpha}\left|\widetilde{c}_{\alpha}^{n-1}(x)\right|
$$

if $\operatorname{dist}\left(U_{\alpha}^{n-1}, \hat{V}_{n-2}\right) \gtrsim 1 / K_{n-1}$. We can choose the same linear subspace $V_{n-2}$ for all $x$ in a ball of size $K_{n-2}$. 
First assume 2.i) holds for a fixed $x$. Then

$$
\left|\int_{\left\{\xi: \operatorname{dist}\left(\xi, \hat{V}_{n-1}\right) \lesssim 1 / K_{n}\right\}} f(\xi) e^{-i x \cdot \xi} d \sigma(\xi)\right| \lesssim K_{n-1}^{2 n-3}\left(\prod_{i=1}^{n-1} \widetilde{c}_{\alpha_{i}}^{n-1}(x)\right)^{1 /(n-1)} .
$$

If $p \geq 2(n-1) /(n-2)$ then we proceed to use the multilinear theory of [1] to estimate:

$$
\begin{aligned}
\int_{B_{2.1}} \mid \int_{\left\{\xi: \operatorname{dist}\left(\xi, \hat{V}_{n-1}\right) \lesssim 1 / K_{n}\right\}} & \left.f(\xi) e^{-i x \cdot \xi} d \sigma(\xi)\right|^{p} d x \\
& \lesssim C\left(K_{n-1}\right) \int_{B_{R}}\left(\prod_{i=1}^{n-1} \widetilde{c}_{\alpha_{i}}^{n-1}(x)\right)^{p /(n-1)} d x,
\end{aligned}
$$

where choices of $\widetilde{c}_{\alpha_{i}}^{n-1}$ of course depend on $x$. Clearly

$$
\begin{aligned}
& \lesssim C\left(K_{n-1}\right) \int_{B_{R}}\left(\prod_{i=1}^{n-1} \sum_{\alpha_{i}} c_{\alpha_{i}}^{n}(x)\right)^{p /(n-1)} d x \\
& \lesssim C\left(K_{n}\right) \sum_{\alpha_{1}, \ldots, \alpha_{n-1}} \int_{B_{R}}\left(\prod_{i=1}^{n-1} c_{\alpha_{i}}^{n}(x)\right)^{p /(n-1)} d x
\end{aligned}
$$

and applying the same arguments as in case 1.i) we obtain

$$
\lesssim C\left(K_{n}\right) R^{\epsilon} \lesssim R^{2 \epsilon}
$$

Thus we assume $p<2(n-1) /(n-2)$. In this case we have

$$
\begin{aligned}
& \oint_{B\left(a, K_{n}\right)}\left|\int_{\left\{\xi: \operatorname{dist}\left(\xi, \hat{V}_{n-1}\right) \lesssim 1 / K_{n}\right\}} f(\xi) e^{-i x \cdot \xi} d \sigma(\xi)\right|^{p} d x \\
& \leq K_{n-1}^{(2 n-3) p} \sum_{\alpha_{1}, \ldots, \alpha_{n-1}} \oint_{B\left(a, K_{n}\right)}\left(\prod_{i=1}^{n-1} \widetilde{c}_{\alpha_{i}}^{n-1}(x)\right)^{p /(n-1)} d x,
\end{aligned}
$$

where the subspace $V_{n-1}$ remains the same for all $x \in B\left(a, K_{n}\right)$. The choice of $\alpha_{1}, \ldots, \alpha_{n-1}$ remains the same only in balls of size $K_{n-1}$, but since the subspace is the same, the caps $U_{\alpha}^{n-1}$ are always chosen from those intersecting the set

$$
\left\{\xi: \operatorname{dist}\left(\xi, \hat{V}_{n-1}\right) \lesssim 1 / K_{n}\right\}
$$

We will exploit multilinearity partially. Consider an individual integral from (2.4) above; since $p<2(n-1) /(n-2)$ we have, by the Hölder inequality,

(2.5) $\oint_{B\left(a, K_{n}\right)}\left(\prod_{i=1}^{n-1} \widetilde{c}_{\alpha_{i}}^{n-1}(x)\right)^{p /(n-1)} d x \lesssim\left(\oint_{B\left(a, K_{n}\right)}\left(\prod_{i=1}^{n-1} \widetilde{c}_{\alpha_{i}}^{n-1}(x)\right)^{2 /(n-2)} d x\right)^{r}$, 
where $r:=\frac{p(n-2)}{2(n-1)}$. This, by the definition of $\widetilde{c}_{\alpha}^{n-1}$, satisfies

$$
\lesssim\left(\oint_{B\left(a, K_{n}\right)}\left(\prod_{i=1}^{n-1} \int\left|\widetilde{T}_{\alpha}^{n-1} f\left(x-y_{i}\right)\right|^{1 /(n-1)} \zeta_{K_{n-1}}\left(y_{i}\right) d y_{i}\right)^{2(n-1) /(n-2)} d x\right)^{r}
$$

Using first the Hölder inequality then Fubini's theorem we have

$$
\begin{aligned}
& \lesssim\left[\oint_{B\left(a, K_{n}\right)}\left(\prod_{i=1}^{n-1} \int\left|\widetilde{T}_{\alpha}^{n-1} f\left(x-y_{i}\right)\right|^{2 /(n-2)} \zeta_{K_{n-1}}\left(y_{i}\right) d y_{i}\right) d x\right]^{r} \\
& \lesssim\left[\int\left(\oint_{B\left(a, K_{n}\right)} \prod_{i=1}^{n-1}\left|\widetilde{T}_{\alpha}^{n-1} f\left(x-y_{i}\right)\right|^{2 /(n-2)} d x\right) \prod_{i=1}^{n-1} \zeta_{K_{n-1}}\left(y_{i}\right) d y_{1} \ldots d y_{n-1}\right]^{r} .
\end{aligned}
$$

Now apply Lemma 2 to the inner integral to obtain

$$
\lesssim K_{n}^{\epsilon}\left[\int\left(\oint_{B\left(a, K_{n}\right)} \prod_{i=1}^{n-1}\left(\sum_{\alpha_{i}}\left|T_{\alpha_{i}}^{n} f\left(x-y_{i}\right)\right|^{2}\right)^{1 /(n-2)} d x\right) \prod_{i=1}^{n-1} \zeta_{K_{n-1}}\left(y_{i}\right) d y_{1} \ldots d y_{n-1}\right]^{r}
$$

where the summation is over all $\alpha_{i}$ such that $U_{\alpha_{i}}^{n} \subset U_{\alpha_{i}}^{n-1}$ and $U_{\alpha_{i}}^{n} \cap \hat{V}_{n-1} \neq \emptyset$. Since $p<2(n-1)(n-2)$, by the Hölder inequality and Fubini's theorem,

$$
\begin{aligned}
& \lesssim K_{n}^{\epsilon+(n-2)(p / 2-1)}\left[\oint_{B\left(a, K_{n}\right)}\left(\prod_{i=1}^{n-1} \int\left(\sum_{\alpha_{i}}\left|T_{\alpha_{i}}^{n} f\left(x-y_{i}\right)\right|^{p}\right) \zeta_{K_{n-1}}\left(y_{i}\right) d y_{i}\right) d x\right]^{1 /(n-1)} \\
& \lesssim K_{n}^{\epsilon+(n-2)(p / 2-1)}\left[\oint_{B\left(a, K_{n}\right)}\left(\sum_{\alpha} \int\left|T_{\alpha}^{n} f(x-y)\right|^{p} \zeta_{K_{n-1}}(y) d y\right)^{n-1} d x\right]^{1 /(n-1)} .
\end{aligned}
$$

From the definition of $c_{\alpha}^{n}$,

$$
\begin{aligned}
& \lesssim K_{n}^{\epsilon+(n-2)(p / 2-1)}\left[\oint_{B\left(a, K_{n}\right)}\left(\sum_{\alpha} \int\left(c_{\alpha}^{n}(x-y)\right)^{p} \zeta_{K_{n-1}}(y) d y\right)^{n-1} d x\right]^{1 /(n-1)} \\
& \lesssim K_{n}^{\epsilon+(n-2)(p / 2-1)}\left[\oint_{B\left(a, K_{n}\right)}\left(\sum_{\alpha}\left(c_{\alpha}^{n}(x)\right)^{p}\right)^{n-1} d x\right]^{1 /(n-1)},
\end{aligned}
$$

where the $\alpha$ in the summation is unrestricted. At this point we use the fact that the sum inside is constant:

$$
\lesssim K_{n}^{\epsilon+(n-2)(p / 2-1)} \oint_{B\left(a, K_{n}\right)} \sum_{\alpha}\left(c_{\alpha}^{n}(x)\right)^{p} d x
$$

Integrating both sides over $B_{2.1}$ we obtain

$$
\begin{aligned}
\int_{B_{2.1}}\left(\prod_{i=1}^{n-1} \widetilde{c}_{\alpha_{i}}^{n-1}(x)\right)^{p /(n-1)} d x & \int_{B_{R}}\left(\sum_{\alpha} \int\left|T_{\alpha}^{n} f(x-y)\right|^{p} \zeta_{K_{n}}(y) d y\right) d x \\
& \lesssim K_{n}^{\epsilon+(n-2)(p / 2-1)} \\
& \lesssim K_{n}^{\epsilon+(n-2)(p / 2-1)} \sum_{\alpha} \int\left(\int_{B_{R}}\left|T_{\alpha}^{n} f(x-y)\right|^{p} d x\right) \zeta_{K_{n}}(y) d y
\end{aligned}
$$


Now we apply rescaling to obtain

$$
\lesssim K_{n}^{(n-2)(1 / 2-1 / p)+(n-1) / p-(n-1)+(n+1) / p+\epsilon} Q_{R}^{p} \lesssim K_{n}^{\epsilon+(n+2) / p-n / 2} Q_{R}^{p} .
$$

Thus we finally get

$$
\int_{B_{2,1}}\left|\int_{\left\{\xi: \operatorname{dist}\left(\xi, \hat{V}_{n-1}\right) \lesssim 1 / K_{n}\right\}} f(\xi) e^{-i x \cdot \xi} d \sigma(\xi)\right|^{p} d x \lesssim C\left(K_{n-1}\right) K_{n}^{\epsilon+(n+2) / p-n / 2} Q_{R}^{p} .
$$

Choosing $K_{n}$ suitably with respect to $K_{n-1}$ shows that $p>2(n+2) / n$ makes this term acceptable. Thus we obtain the condition $p>\min (2 n /(n-1), 2(n+2) / n)$.

Next we proceed to handle 2.ii) in a similar way. We define $\widetilde{c}^{n-2}$ from intersections of caps with a $1 / K_{n-1}$ neighborhood of our subspace and $c^{n-2}$ from full caps. In our analysis $\widetilde{c}^{n-1}$ will then be replaced by $\widetilde{c}^{n-1}$, and $c^{n}$ by $c^{n-1}$. Continuing this process gives the condition

$$
p>2 \min \left(\frac{k}{k-1}, \frac{2 n-k+1}{2 n-k-1}\right), \quad \text { for all } \quad 2 \leq k \leq n,
$$

which gives (1.3). Here $p$ should be greater than the minimum for all values of $2 \leq k \leq n$. Thus the condition is that $p$ should be greater than the maximum of these minima. When $n \equiv 0 \bmod 3$ this maximum is attained only at the value $k=2 n / 3$ and it comes from the second term, thus has the value $2(4 n+3) /(4 n-3)$. The value of the first term, then, is $4 n /(2 n-3)$, which is strictly greater than $(8 n+6) /(4 n-3)$. This is what allows us to improve the exponent when $n \equiv 0 \bmod 3$. For the particular case of $n=6$ we have

$$
p>2 \min \left(\frac{k}{k-1}, \frac{13-k}{11-k}\right), \quad \text { for all } \quad 2 \leq k \leq 6 .
$$

For $k=6,5$ the minimum comes from the first term, for other $k$ from the second term. The maximum of these minima comes from $k=4$ and is $18 / 7$. For $k=4$ the first term gives $8 / 3>18 / 7$ and in our refined analysis we shall exploit this.

\section{Refined analysis}

From the analysis of the last section, for a fixed $x \in B(0, R)$ we can write

$$
\begin{aligned}
|T f(x)| \leq & C\left(K_{6}\right) \max _{i_{1}, \ldots, i_{6}}\left(c_{i_{1}}^{6}(x) \ldots c_{i_{6}}^{6}(x)\right)^{1 / 6} \\
& +\sum_{m=2}^{5} C\left(K_{m}\right) \max _{V_{m}} \prod_{k=1}^{m}\left(\widetilde{c}_{i_{k}}^{m}(x)\right)^{1 / m}+C\left(K_{1}\right) \max _{\alpha} c_{\alpha}^{2}(x) .
\end{aligned}
$$

Here by $i_{k}$ we denote a cap of radius $1 / K_{m}$ in the $m$-linear term, $V_{m}$ denotes an $m$-dimensional subspace and $\hat{V}_{m}$ the image of $V_{m} \cap S^{5}$ under the Gauss map. Our idea is, as among these terms the 4-linear gives the worst exponent, iterating this decomposition for smaller caps in that term we may obtain an improvement. 
We will also iterate the decomposition for the linear, bilinear, and trilinear terms. To execute this idea we replace the terms we want to further decompose as follows. Let $L_{m}$ denote the caps $j$ of size $1 / K_{m+1}$ such that $j \cap \hat{V}_{m} \neq \emptyset$. The calculation we did to bound (2.5) using Lemma 2 without the use of the Hölder inequality to raise the exponent to $p$ in $(2.6)$ gives

$$
\left[\oint_{B\left(a, K_{5}\right)} \prod_{k=1}^{4}\left(\widetilde{c}_{i_{k}}^{4}(x)\right)^{2 / 3} d x\right]^{3 / 8} \lesssim K_{5}^{\epsilon}\left(\sum_{j \in L_{4}}\left(c_{j}^{5}(x)\right)^{2}\right)^{1 / 2}
$$

where, on the right-hand side, $x \in B\left(a, K_{5}\right)$. Using this and the fact that $\widetilde{c}^{4}(x)$ are constant on balls of radius $K_{4}$ we may write

$$
\prod_{k=1}^{4}\left(\widetilde{c}_{i_{k}}^{4}(x)\right)^{1 / 4}=\phi_{4} \cdot\left(\sum_{j \in L_{4}}\left(c_{j}^{5}(x)\right)^{2}\right)^{1 / 2},
$$

where $\phi_{4}$ is constant on balls of radius 1 and satisfies

$$
\left(\oint_{B\left(a, K_{5}\right)}\left(\phi_{4}\right)^{8 / 3}\right)^{3 / 8} \lesssim K_{5}^{\epsilon}
$$

Notice that if sum on the right-hand side of (3.2) is zero then the left-hand side is also zero, hence the function $\phi_{4}$ can be constructed simply by dividing the lefthand term by the sum on the right when sum is not zero, and by setting it to zero when it is. For the bilinear and the trilinear terms we have similarly

$$
\begin{gathered}
{\left[\oint_{B\left(a, K_{4}\right)} \prod_{k=1}^{3} \widetilde{c}_{i_{k}}^{3}(x) d x\right]^{1 / 3} \lesssim K_{4}^{\epsilon}\left(\sum_{j \in L_{3}}\left(c_{j}^{4}(x)\right)^{2}\right)^{1 / 2}} \\
{\left[\oint_{B\left(a, K_{3}\right)} \prod_{k=1}^{2}\left(\widetilde{c}_{i_{k}}^{2}(x)\right)^{2} d x\right]^{1 / 4} \lesssim K_{3}^{\epsilon}\left(\sum_{j \in L_{2}}\left(c_{j}^{3}(x)\right)^{2}\right)^{1 / 2} .}
\end{gathered}
$$

So we can find $\phi_{3}$ and $\phi_{2}$ that are constant on balls of unit size that satisfy

$$
\begin{gathered}
\prod_{k=1}^{3}\left(\widetilde{c}_{i_{k}}^{3}(x)\right)^{1 / 3}=\phi_{3} \cdot\left(\sum_{j \in L_{3}}\left(c_{j}^{4}(x)\right)^{2}\right)^{1 / 2}, \\
\prod_{k=1}^{2}\left(\widetilde{c}_{i_{k}}^{2}(x)\right)^{1 / 2}=\phi_{2} \cdot\left(\sum_{j \in L_{2}}\left(c_{j}^{3}(x)\right)^{2}\right)^{1 / 2}, \\
\left(\oint_{B\left(a, K_{4}\right)}\left(\phi_{3}\right)^{3}\right)^{1 / 3} \lesssim K_{4}^{\epsilon}, \quad\left(\oint_{B\left(a, K_{3}\right)}\left(\phi_{2}\right)^{4}\right)^{1 / 4} \lesssim K_{3}^{\epsilon} .
\end{gathered}
$$

Thus we can write

$$
\begin{aligned}
|T f(x)| \leq & C\left(K_{6}\right) \max _{i_{1}, \ldots, i_{6}}\left(c_{i_{1}}^{6}(x) \ldots c_{i_{6}}^{6}(x)\right)^{1 / 6}+C\left(K_{5}\right) \max _{V_{5}} \prod_{k=1}^{5}\left(\widetilde{c}_{i_{k}}^{5}(x)\right)^{1 / 5} \\
& +\sum_{m=2}^{4} K_{m+1}^{\epsilon} \phi_{m} \cdot \max _{V_{m}}\left(\sum_{j \in L_{m}}\left(c_{j}^{m+1}\right)^{2}\right)^{1 / 2}+C\left(K_{1}\right) \max _{\alpha} c_{\alpha}^{2}(x) .
\end{aligned}
$$


We shall iterate our decomposition for these caps $j$. We now describe this in a general fashion. Let $\tau$ be a cap of radius $\delta$. By first scaling to the unit scale, then applying the decomposition, then scaling back we get

$$
\begin{aligned}
|T f(x)| \leq & C\left(K_{6}\right) \max _{i_{1}, \ldots, i_{6}}\left(c_{\tau_{1}}^{6}(x) \ldots c_{\tau_{6}}^{6}(x)\right)^{1 / 6}+C\left(K_{5}\right) \max _{V_{5}} \prod_{k=1}^{5}\left(\widetilde{c}_{\tau_{k}}^{5}(x)\right)^{1 / 5} \\
& +\sum_{m=2}^{4} K_{m+1}^{\epsilon} \phi_{\tau_{m}} \cdot \max _{V_{m}}\left(\sum_{j \in L_{m}}\left(c_{\eta}^{m+1}(x)\right)^{2}\right)^{1 / 2}+C\left(K_{1}\right) \max _{\alpha} c_{\tau_{\alpha}}^{2}(x) .
\end{aligned}
$$

Here, similar to what we have above by $\tau_{k}$ we denote caps that are of radius $1 / K_{m}$ in the $m$-linear term, and the notation $\eta$ denotes caps of radius $1 / K_{m+1}$ in the $m$-linear term. Furthermore we have $\phi_{\tau_{m}}$ constant on boxes $\tau^{\prime}$ dual to cap $\tau$ and for boxes $K_{m+1} \tau^{\prime}$,

$$
\oint_{B} \phi_{\tau_{m}}^{8 / 3} \lesssim K_{m+1}^{\epsilon}
$$

These two are simple consequences of rescaling.

We iterate this decomposition except for the 6-linear and 5-linear terms in the main decomposition. We clarify several points that arises from application of this process. First of all from the second step onwards we actually apply the decomposition not to expressions of type $T f_{\tau}$ but to $c_{\tau}$. This is a simple issue to handle, and the right-hand side remains the same. To see this notice that all terms on the right-hand side are already constant on balls exceeding the size of averaging we need to pass from $T_{\tau}$ to $c_{\tau}$. Second, as we iterate, we will need to multiply functions $\phi_{\tau_{m}}$ arising on each step of the iteration. To investigate what happens in this case, let $\phi_{\tau_{k}}$ and $\phi_{\eta_{l}}$ be such functions arising in consecutive steps. Thus $\phi_{\tau_{k}}$ is constant on boxes $\tau^{\prime}$ dual to the cap $\tau$, and $\phi_{\eta}$ constant on boxes $\eta^{\prime}$ dual to the cap $\eta$. As the $\eta$ arise when we decompose $\tau$, if we let $\tau$ be a $\delta$ cap, $\eta$ is a $\delta / K_{l+1}$ cap. Now let $B$ be a $K_{l+1} \eta^{\prime}$ box. One can, of course, decompose this box into $\eta^{\prime}$ boxes $B_{\alpha}$; thus, since $\phi_{\eta_{l}}^{8 / 3}$ is comparable to a constant on a $B_{\alpha}$ box,

$$
\left.\int_{B} \phi_{\tau_{k}}^{8 / 3} \phi_{\eta_{l}}^{8 / 3} \lesssim \sum_{\alpha} \phi_{\eta_{l}}^{8 / 3}\right|_{B_{\alpha}} \int_{B_{\alpha}} \phi_{\tau_{k}}^{8 / 3}
$$

where we use the expression

$$
\left.\phi_{\eta_{l}}^{8 / 3}\right|_{B_{\alpha}}
$$

to denote evaluation of $\phi_{\eta_{l}}^{8 / 3}$ on an arbitrary point of $B_{\alpha}$. The direction of a $\tau^{\prime}$ box differs from that of a $\eta^{\prime}$ only by an angle of $\delta$, thus when it is decomposed, the average over the larger box should be no greater than the maximum of the averages over the smaller boxes. Hence

$$
\oint_{B_{\alpha}} \phi_{\tau_{k}}^{8 / 3} \lesssim K_{k+1}^{\epsilon}
$$


so we have

$$
\begin{aligned}
\left.\sum_{\alpha} \phi_{\eta_{l}}^{8 / 3}\right|_{B_{\alpha}} \int_{B_{\alpha}} \phi_{\tau_{k}}^{8 / 3} & \lesssim \sum_{\alpha} \int_{B_{\alpha}} \phi_{\eta_{l}}^{8 / 3} \oint_{B_{\alpha}} \phi_{\tau_{k}}^{8 / 3} \lesssim K_{k+1}^{\epsilon} \sum_{\alpha} \int_{B_{\alpha}} \phi_{\eta_{l}}^{8 / 3} \\
& \lesssim K_{k+1}^{\epsilon} \int_{B} \phi_{\eta_{l}}^{8 / 3} \lesssim K_{k+1}^{\epsilon} K_{l+1}^{\epsilon}|B|
\end{aligned}
$$

This also shows that emerging cross terms will not lead to any problem in the iteration process as the losses $K_{m+1}^{\epsilon}$ are proportionate to the size of the caps $\tau_{m}$. That is, if we divide a cap into larger caps, the number of steps we iterate our decomposition increases, but since the losses incurred at each step are smaller this does not lead to any problem. After this investigation we are ready to state the final situation after iterating the decomposition,

$$
\begin{aligned}
& |T f| \leq R^{\epsilon} \max _{R^{-1 / 2}<\delta<1} \max _{E_{\delta}}\left[\sum_{\tau \in E_{\delta}}\left(\phi_{\tau} \max _{\tau_{1}, \ldots, \tau_{6}}\left(c_{\tau_{1}}^{6} \ldots c_{\tau_{6}}^{6}\right)^{1 / 6}\right)^{2}\right]^{1 / 2} \\
& +R^{\epsilon} \max _{R^{-1 / 2}<\delta<1} \max _{E_{\delta}}\left[\sum_{\tau \in E_{\delta}}\left(\phi_{\tau}\left(\max _{V_{4}} \prod_{k=1}^{5} \widetilde{c}_{\tau_{k}}^{5}\right)^{1 / 5}\right)^{2}\right]^{1 / 2}+R^{\epsilon} \max _{E_{R^{-1 / 2}}}\left[\sum_{\tau \in E}\left(\phi_{\tau} c_{\tau}\right)^{2}\right]^{1 / 2},
\end{aligned}
$$

where

1) in all terms $E_{\delta}$ is a collection of $\delta$ caps, with cardinality $\delta^{-3}$;

2 ) in the $m$-linear term $\tau_{j} \subset \tau$ are caps of size $\delta / K_{m}$ satisfying $\tau_{j} \cap \hat{V}_{m-1} \neq \emptyset$ and the linear independence condition;

3) for $B$ a $\tau^{\prime}$ box we have

$$
\oint_{B} \phi_{\tau}^{8 / 3}<R^{\epsilon}
$$

We shall estimate each of the terms above. For each of the $m$-linear terms with $m>1$ we proceed as follows. We first estimate the term in the $L^{p}$ space with the exponent given by $(2.9)$ for $k=m$. Then we estimate at $L^{18 / 7}$, which is the maximum of the exponents given by (2.9) exactly in the same fashion. Using this, we make a refined estimate using pigeonholing at the exponent 18/7 in two different ways to obtain a small gain. Interpolation with the estimate at the exponent given by (2.9) will determine the small improvement to the exponent $18 / 7$. For the linear term the process is similar but simpler. We start by estimating the 6 -linear term.

\subsection{Estimates on the 6-linear term}

We consider the term

$$
\max _{E_{\delta}}\left[\sum_{\tau \in E_{\delta}}\left(\phi_{\tau} \max _{\tau_{1}, \ldots, \tau_{6}}\left(c_{\tau_{1}}^{6} \ldots c_{\tau_{6}}^{6}\right)^{1 / 6}\right)^{2}\right]^{1 / 2} .
$$

The inner maximum has no importance and we can fix $c_{\tau_{i}}^{6}$. We wish to exploit the fact that the Fourier transform of a function supported on a small cap is roughly 
constant on a tube dual to this cap. To make this precise we shall need some more notation. Recall that we chose a function $\eta \in \mathcal{S}\left(\mathbb{R}^{n}\right)$ with $\widehat{\eta}(x)=1$ on $B(0,1)$ and $\widehat{\eta}(x)=0$ outside $B(0,2)$. Rescale this function to obtain a function $v_{\tau_{i}}$ adapted to a tube dual to the cap $\tau_{i}$. Similarly obtain $\beta_{\tau_{i}}$ by rescaling $\zeta$. With $v_{\tau_{i}}, \beta_{\tau_{i}}$ we define $b_{\tau_{i}}^{6}$ in the same way that $c_{\tau_{i}}^{6}$ was defined on page 1019 . Then

$$
\left|T f_{\tau_{i}}\right| \lesssim b_{\tau_{i}}^{6}
$$

Convolving both sides with $\zeta_{\tau_{i}}$, and using the fact that the $b_{\tau_{i}}^{6}$ are actually constant on balls of the size at which this averaging occurs, we obtain

$$
c_{\tau_{i}}^{6} \lesssim b_{\tau_{i}}^{6}
$$

Thus we can estimate (3.3) by

$$
\max _{E_{\delta}}\left[\sum_{\tau \in E_{\delta}}\left(\phi_{\tau}\left(b_{\tau_{1}}^{6} \ldots b_{\tau_{6}}^{6}\right)^{1 / 6}\right)^{2}\right]^{1 / 2} .
$$

Assume $|f| \leq 1$. We have

$$
\int_{B_{R}}\left(b_{\tau_{1}}^{6} \ldots b_{\tau_{6}}^{6}\right)^{2 / 5}=\int_{B_{R}}\left(\prod_{i=1}^{6} \int\left|T f_{\tau_{i}}(x-y)\right|_{\delta / K_{6}}^{1 / 6} \beta_{\delta / K_{6}}(y) d y\right)^{12 / 5} d x
$$

and by the Hölder inequality

$$
\begin{aligned}
& \lesssim \int_{B_{R}}\left(\prod_{i=1}^{6} \int\left|T f_{\tau_{i}}(x-y)\right|^{2 / 5} \beta_{\delta / K_{6}}(y) d y\right) d x \\
& =\int_{B_{R}}\left(\int \prod_{i=1}^{6}\left|T f_{\tau_{i}}\left(x-y_{i}\right)\right|^{2 / 5} \beta_{\delta / K_{6}}\left(y_{i}\right) d y_{1} \ldots d y_{6}\right) d x,
\end{aligned}
$$

which, by Fubini's theorem, becomes

$$
=\int\left(\int_{B_{R}} \prod_{i=1}^{6}\left|T f_{y_{i}, \tau_{i}}(x)\right|^{2 / 5} d x\right) \prod_{i=1}^{6} \beta_{\delta / K_{6}}\left(y_{i}\right) d y_{1} \ldots d y_{6} .
$$

Of course, $f_{y_{i}}$ are modulations of $f$. Now rescaling the inside integral to obtain functions $\left|g_{y_{i}}\right| \leq 1$ and caps $U_{1}, \ldots, U_{6}$ of size $1 / K_{6}$ satisfying the linear independence condition we have

$$
\lesssim \delta^{5} \int\left(\int_{B_{R}} \prod_{i=1}^{6}\left|T g_{y_{i}, U_{i}}(x)\right|^{2 / 5} d x\right) \prod_{i=1}^{6} \beta_{\delta / K_{6}}\left(y_{i}\right) d y_{1} \ldots d y_{6} .
$$

Thus the multilinear theory of [1] applies to yield

$$
\lesssim \delta^{5} R^{\epsilon}
$$


With this in hand we proceed to estimate at the exponent $12 / 5$ given by $(2.9)$. Using the Hölder inequality we have

$$
\begin{aligned}
{\left[\sum_{\tau \in E_{\delta}}\left(\phi_{\tau}\left(b_{\tau_{1}}^{6} \ldots b_{\tau_{6}}^{6}\right)^{1 / 6}\right)^{2}\right]^{1 / 2} } & \leq\left|E_{\delta}\right|^{1 / 12}\left[\sum_{\tau \in E_{\delta}}\left(\phi_{\tau}\left(b_{\tau_{1}}^{6} \ldots b_{\tau_{6}}^{6}\right)^{1 / 6}\right)^{12 / 5}\right]^{5 / 12} \\
& \lesssim \delta^{-1 / 4}\left[\sum_{\tau \in E_{\delta}}\left(\phi_{\tau}\left(b_{\tau_{1}}^{6} \ldots b_{\tau_{6}}^{6}\right)^{1 / 6}\right)^{12 / 5}\right]^{5 / 12}
\end{aligned}
$$

Now $\tau$ ranges over a full partition into $\delta$ caps of $S$, and does not depend on the particular choice of $E_{\delta}$. Now let $B$ stand for a $\tau^{\prime}$ box. Since the $b_{\tau_{i}}^{6}$ are constant on $\tau_{i}^{\prime}$ boxes, we have

$$
\begin{aligned}
\int_{B_{R}} & \left.\left(\phi_{\tau}\left(b_{\tau_{1}}^{6} \ldots b_{\tau_{6}}^{6}\right)^{1 / 6}\right)^{12 / 5} \lesssim \sum_{B}\left(b_{\tau_{1}}^{6} \ldots b_{\tau_{6}}^{6}\right)^{2 / 5}\right|_{B}\left(\int_{B} \phi_{\tau}^{12 / 5}\right) \\
& \lesssim \sum_{B}\left(\int_{B}\left(b_{\tau_{1}}^{6} \ldots b_{\tau_{6}}^{6}\right)^{2 / 5}\right)\left(\oint_{B} \phi_{\tau}^{12 / 5}\right) \lesssim R^{\epsilon} \int_{B_{R}}\left(b_{\tau_{1}}^{6} \ldots b_{\tau_{6}}^{6}\right)^{2 / 5} \lesssim R^{\epsilon} \delta^{5}
\end{aligned}
$$

Using this we finally obtain

$$
\|(3.3)\|_{L^{12 / 5}\left(B_{R}\right)} \lesssim R^{\epsilon} \delta^{-1 / 4}
$$

On the other hand applying the same process and using the fact $b_{\tau_{i}}^{6} \lesssim \delta^{5}$ to reduce the exponent yields

$$
\|(3.3)\|_{L^{18 / 7}\left(B_{R}\right)} \lesssim R^{\epsilon}
$$

Now we begin the finer estimates. Let $0<\lambda<1$ and define

$$
g_{\tau, \lambda}=g_{\tau} 1_{\left\{g_{\tau} \sim \lambda \delta^{5}\right\}}, \quad \text { where } \quad g_{\tau}=\left(b_{\tau_{1}}^{6} \ldots b_{\tau_{6}}^{6}\right)^{1 / 6} .
$$

Then

$$
\int_{B_{R}} g_{\tau, \lambda}^{18 / 7}<\left(\lambda \delta^{5}\right)^{18 / 7-12 / 5} \int_{B_{R}} g_{\tau, \lambda}^{12 / 5} \lesssim R^{\epsilon} \lambda^{6 / 35} \delta^{41 / 7}
$$

Using this

$$
\left[\int_{B_{R}} \max _{E_{\delta}}\left(\sum_{\tau \in E_{\delta}}\left(\phi_{\tau} g_{\tau, \lambda}\right)^{2}\right)^{9 / 7}\right]^{7 / 18} \lesssim R^{\epsilon} \lambda^{1 / 15}
$$

We pigeonhole one more time. Let $1 \leq \mu<\infty$ and decompose

$$
\phi_{\tau}=\sum_{\mu \text { dyadic }} \phi_{\tau, \mu}
$$

where

$$
\phi_{\tau, \mu}=\phi_{\tau} 1_{\left\{\phi_{\tau} \sim \mu\right\}}, \quad \phi_{\tau, 1}=\phi_{\tau} 1_{\left\{\phi_{\tau} \leq 1\right\}} .
$$

Then we have

$$
\oint_{B} \phi_{\tau, \mu}^{18 / 7} \leq \mu^{-2 / 21} \oint_{B} \phi_{\tau, \mu}^{8 / 3} \lesssim R^{\epsilon} \mu^{-2 / 21}
$$


and

$$
\left[\int_{B_{R}} \max _{E_{\delta}}\left(\sum_{\tau \in E_{\delta}}\left(\phi_{\tau, \mu} g_{\tau, \lambda}\right)^{2}\right)^{9 / 7}\right]^{7 / 18} \lesssim R^{\epsilon} \lambda^{1 / 15} \mu^{-1 / 27} .
$$

We now estimate the left hand side of the inequality above in a different way. Clearly we have

$$
\max _{E_{\delta}}\left(\sum_{\tau \in E_{\delta}}\left(\phi_{\tau, \mu} g_{\tau, \lambda}\right)^{2}\right)^{1 / 2} \leq \mu\left(\sum_{\tau} g_{\tau, \lambda}^{2}\right)^{1 / 2}
$$

Now $\tau$ ranges over a full partition into $\delta$ caps of the surface $S$. We shall write the right-hand side as convolutions of measures with tubes, and apply Kakeya maximal function estimates. Since the separation between the directions of the caps $\tau$ and $\tau_{i}$ are small we have

$$
\left(b_{\tau_{i}}^{6}\right)^{1 / 6} \lesssim\left(b_{\tau_{i}}^{6}\right)^{1 / 6} *\left(\delta^{7} 1_{\tau^{\prime}}\right) .
$$

Hence

$$
g_{\tau} \lesssim \int\left(\prod_{i=1}^{6}\left(b_{\tau_{i}}^{6}\right)^{1 / 6} * \delta^{7} 1_{\tau^{\prime}}\right)(z)\left(\delta^{7} 1_{\tau^{\prime}}\right)(x-z) d z \lesssim \int \omega(z)\left(\delta^{7} 1_{\tau^{\prime}}\right)(x-z) d z .
$$

By the definition of $g_{\tau, \lambda}$ and (3.4) we have

$$
g_{\tau, \lambda}^{2} \lesssim \omega^{2} 1_{\left\{\omega \gtrsim \lambda \delta^{5}\right\}}
$$

However since the function $\omega$ is constant on tubes dual to $\tau$ we can write

$$
g_{\tau, \lambda}^{2} \lesssim \delta^{7} \int\left(\omega^{2} 1_{\left\{\omega \gtrsim \lambda \delta^{5}\right\}}\right)(z) 1_{\tau^{\prime}}(x-z) d z .
$$

We wish to replace the expression

$$
\left(\omega^{2} 1_{\left\{\omega \gtrsim \lambda \delta^{5}\right\}}\right)(z) d z
$$

with a constant multiple of a probability measure, from which we will pass to the Kakeya maximal function. To this end we estimate the total mass of this measure.

By Chebyshev's inequality,

$$
\int_{B_{R}} \omega^{2} 1_{\left\{\omega \gtrsim \lambda \delta^{5}\right\}}(x) d x \lesssim\left(\frac{1}{\lambda \delta^{5}}\right)^{2 / 5} \int_{B_{R}} \omega^{12 / 5}(x) d x
$$

which is

$$
\begin{aligned}
& \lesssim\left(\frac{1}{\lambda \delta^{5}}\right)^{2 / 5} \int\left(\int_{B_{R}}\left(\prod_{i=1}^{6} b_{\tau_{i}}^{6}\left(x-z_{i}\right)\right)^{2 / 5} d x\right)\left(\prod_{i=1}^{6}\left(\delta^{7} 1_{\tau^{\prime}}\right)\left(z_{i}\right)\right) d z_{1} \cdots d z_{6} \\
& \lesssim R^{\epsilon} \lambda^{-2 / 5} \delta^{3} .
\end{aligned}
$$


This puts us in a position to bring into play the Kakeya maximal function estimate of Wolff, for we have

$$
g_{\tau, \lambda}^{2} \lesssim R^{\epsilon} \delta^{10} \lambda^{-2 / 5} \int 1_{\tau^{\prime}}(x-y) d \mu_{\tau},
$$

where $d \mu_{\tau}$ is a probability distribution. From this and convexity we have

$$
\begin{aligned}
\left\|\max _{E_{\delta}}\left(\sum_{\tau \in E_{\delta}}\left(\phi_{\tau, \mu} g_{\tau, \lambda}\right)^{2}\right)^{1 / 2}\right\|_{L^{18 / 7}\left(B_{R}\right)} & \leq R^{\epsilon} \mu \delta^{5} \lambda^{-1 / 5}\left\|\left(\sum_{\tau} 1_{\tau^{\prime}}\left(x-y_{\tau}\right)\right)^{1 / 2}\right\|_{L^{18 / 7\left(B_{R}\right)}} \\
& =R^{\epsilon} \mu \delta^{5} \lambda^{-1 / 5}\left\|\left(\sum_{\tau} 1_{\tau^{\prime}}\left(x-y_{\tau}\right)\right)\right\|_{L^{9 / 7\left(B_{R}\right)}}^{1 / 2},
\end{aligned}
$$

where $y_{\tau}$ is a choice of points in $\mathbb{R}^{6}$. Now we are ready to apply Wolff's Kakeya estimate from [13]. For $\delta$-separated $\delta$-tubes $T$ this estimate gives

$$
\left\|\sum_{T} \chi_{T}\right\|_{L^{4 / 3}} \lesssim \delta^{-1 / 2-} .
$$

Interpolating this with the trivial estimate at $L^{1}$ yields

$$
\left\|\sum_{T} \chi_{T}\right\|_{L^{9 / 7}} \lesssim \delta^{-4 / 9-} .
$$

We rescale the estimate since the sizes of our tubes vary. This gives for our tubes

$$
\left\|\sum_{\tau} 1_{\tau^{\prime}}\left(x-y_{\tau}\right)\right\|_{L^{9 / 7}\left(B_{R}\right)}^{1 / 2} \lesssim \delta^{-44 / 9}
$$

Thus finally

$$
\left\|\max _{E_{\delta}}\left(\sum_{\tau \in E_{\delta}}\left(\phi_{\tau, \mu} g_{\tau, \lambda}\right)^{2}\right)^{1 / 2}\right\|_{L^{18 / 7}\left(B_{R}\right)} \lesssim R^{\epsilon} \mu \delta^{1 / 9} \lambda^{-1 / 5} \lesssim R^{\epsilon} \mu \delta^{1 / 9} \lambda^{-9 / 5} .
$$

Combining our estimates we obtain

$$
\begin{aligned}
\left\|\max _{E_{\delta}}\left(\sum_{\tau \in E_{\delta}}\left(\phi_{\tau} g_{\tau}\right)^{2}\right)^{1 / 2}\right\|_{L^{12 / 5}\left(B_{R}\right)} & \lesssim R^{\epsilon} \delta^{-1 / 4}, \\
\left\|\max _{E_{\delta}}\left(\sum_{\tau \in E_{\delta}}\left(\phi_{\tau, \mu} g_{\tau, \lambda}\right)^{2}\right)^{1 / 2}\right\|_{L^{18 / 7}\left(B_{R}\right)} & \lesssim R^{\epsilon} \min \left(\mu \lambda^{-9 / 5} \delta^{1 / 9}, \lambda^{1 / 15} \mu^{-1 / 27}\right) \\
& \lesssim R^{\epsilon} \delta^{1 /(9 \cdot 28)} .
\end{aligned}
$$

Interpolating these gives the small improvement

$$
\frac{2}{735} \text {. }
$$




\subsection{Estimates on the 5-linear term}

We consider the term

$$
\max _{E_{\delta}}\left[\sum_{\tau \in E_{\delta}}\left(\phi_{\tau}\left(\max _{V_{4}} \prod_{k=1}^{5} \widetilde{c}_{\tau_{k}}^{5}\right)^{1 / 5}\right)^{2}\right]^{1 / 2} .
$$

This, as above, can be estimated by

$$
\max _{E_{\delta}}\left[\sum_{\tau \in E_{\delta}}\left(\phi_{\tau}\left(\max _{V_{4}} \prod_{k=1}^{5} \widetilde{b}_{\tau_{k}}^{5}\right)^{1 / 5}\right)^{2}\right]^{1 / 2}
$$

Taking the maximum over all $V_{4}$ does not make any difference for our estimates since our estimates will remain the same for all $V_{4}$. Thus fix $V_{4}$. Assume $|f| \leq 1$. Using rescaling as in 6-linear case one obtains

$$
\int_{B_{R}}\left(\prod_{k=1}^{5} \widetilde{b}_{\tau_{k}}^{5}\right)^{1 / 2} \leq \delta^{11 / 2} R^{\epsilon}
$$

With this we proceed as in 6-linear case. By the Hölder inequality,

$$
\begin{aligned}
{\left[\sum_{\tau \in E_{\delta}}\left(\phi_{\tau}\left(\prod_{k=1}^{5} \tilde{b}_{\tau_{k}}^{5}\right)^{1 / 5}\right)^{2}\right]^{1 / 2} } & \leq\left|E_{\delta}\right|^{1 / 10}\left[\sum_{\tau}\left(\phi_{\tau}\left(\prod_{k=1}^{5} \widetilde{b}_{\tau_{k}}^{5}\right)^{1 / 5}\right)^{5 / 2}\right]^{2 / 5} \\
& \leq \delta^{-3 / 10}\left[\sum_{\tau}\left(\phi_{\tau}\left(\prod_{k=1}^{5} \widetilde{b}_{\tau_{k}}^{5}\right)^{1 / 5}\right)^{5 / 2}\right]^{2 / 5}
\end{aligned}
$$

Now $\tau$ ranges over a full partition into $\delta$ caps of $S$, and does not depend on the particular choice of $E_{\delta}$. Now let $B$ stand for a $\tau^{\prime}$ box. Since the $\widetilde{b}_{\tau_{k}}^{5}$ are constant on $\tau_{i}^{\prime}$ boxes, we have

$$
\begin{aligned}
\int_{B_{R}} & \left.\phi_{\tau}\left(\prod_{k=1}^{5} \widetilde{b}_{\tau_{k}}^{5}\right)^{1 / 2} \lesssim \sum_{B}\left(\prod_{k=1}^{5} \widetilde{b}_{\tau_{k}}^{5}\right)^{1 / 2}\right|_{B}\left(\int_{B} \phi_{\tau}^{5 / 2}\right) \\
& \lesssim \sum_{B}\left(\int_{B}\left(\prod_{k=1}^{5} \widetilde{b}_{\tau_{k}}^{5}\right)^{1 / 2}\right)\left(\oint_{B} \phi_{\tau}^{5 / 2}\right) \lesssim R^{\epsilon} \int_{B_{R}}\left(\prod_{k=1}^{5} \widetilde{b}_{\tau_{k}}^{5}\right)^{1 / 2} \lesssim R^{\epsilon} \delta^{11 / 2} .
\end{aligned}
$$

Using this we finally obtain

$$
\|(3.5)\|_{L^{5 / 2}\left(B_{R}\right)} \lesssim R^{\epsilon} \delta^{-1 / 10} .
$$

On the other hand the same process yields

$$
\|(3.5)\|_{L^{18 / 7}\left(B_{R}\right)} \lesssim R^{\epsilon} .
$$

Now we proceed to finer estimates. Now let

$$
g_{\tau, \lambda}=g_{\tau} 1_{\left\{g_{\tau} \sim \lambda \delta^{5}\right\}} \quad \text { where } \quad g_{\tau}=\left(\prod_{k=1}^{5} \widetilde{b}_{\tau_{k}}^{5}\right)^{1 / 5} .
$$


Then, by (3.6),

$$
\int_{B_{R}} g_{\tau, \lambda}^{18 / 7}<\left(\lambda \delta^{5}\right)^{18 / 7-5 / 2} \int_{B_{R}} g_{\tau, \lambda}^{5 / 2} \lesssim R^{\epsilon} \lambda^{1 / 14} \delta^{41 / 7} .
$$

Using this,

$$
\left[\int_{B_{R}} \max _{E_{\delta}}\left(\sum_{\tau \in E_{\delta}}\left(\phi_{\tau} g_{\tau, \lambda}\right)^{2}\right)^{9 / 7}\right]^{7 / 18} \lesssim R^{\epsilon} \lambda^{1 / 36}
$$

Decompose $\phi_{\tau}$ exactly as before. Then we have

$$
\left[\int_{B_{R}} \max _{E_{\delta}}\left(\sum_{\tau \in E_{\delta}}\left(\phi_{\tau, \mu} g_{\tau, \lambda}\right)^{2}\right)^{9 / 7}\right]^{7 / 18} \lesssim R^{\epsilon} \lambda^{1 / 36} \mu^{-1 / 27} .
$$

Now we estimate

$$
\max _{E_{\delta}}\left(\sum_{\tau \in E_{\delta}}\left(\phi_{\tau, \mu} g_{\tau, \lambda}\right)^{2}\right)^{1 / 2}
$$

using the Kakeya maximal function as in the 6-linear case. Clearly

$$
\max _{E_{\delta}}\left(\sum_{\tau \in E_{\delta}}\left(\phi_{\tau, \mu} g_{\tau, \lambda}\right)^{2}\right)^{1 / 2} \leq \mu\left(\sum_{\tau} g_{\tau, \lambda}^{2}\right)^{1 / 2}
$$

where $\tau$ ranges over a full partition into $\delta$ caps of the surface $S$.

$$
\left(\widetilde{b}_{\tau_{k}}^{5}\right)^{1 / 5} \lesssim\left(\widetilde{b}_{\tau_{k}}^{5}\right)^{1 / 5} *\left(\delta^{7} 1_{\tau^{\prime}}\right) .
$$

Hence

$$
g_{\tau} \lesssim \int\left(\prod_{k=1}^{5}\left(\widetilde{b}_{\tau_{i}}^{5}\right)^{1 / 5} * \delta^{7} 1_{\tau^{\prime}}\right)(z)\left(\delta^{7} 1_{\tau^{\prime}}\right)(x-z) d z \lesssim \int \omega(z)\left(\delta^{7} 1_{\tau^{\prime}}\right)(x-z) d z
$$

SO

$$
g_{\tau, \lambda}^{2} \lesssim \delta^{7} \int\left(\omega^{2} 1_{\left\{\omega \gtrsim \lambda \delta^{5}\right\}}\right)(z) 1_{\tau^{\prime}}(x-z) d z
$$

We have

$$
\int_{B_{R}} \omega^{2} 1_{\left\{\omega \gtrsim \lambda \delta^{5}\right\}}(x) d x \lesssim\left(\frac{1}{\lambda \delta^{5}}\right)^{1 / 2} \int_{B_{R}} \omega^{5 / 2}(x) d x
$$

which, by (3.6), yields

$$
\begin{aligned}
& \lesssim\left(\frac{1}{\lambda \delta^{5}}\right)^{1 / 2} \int_{B_{R}}\left(\int\left(\prod_{k=1}^{5} \widetilde{b}_{\tau_{i}}^{5}\left(x-z_{i}\right)\right)^{1 / 2} d x\right)\left(\prod_{k=1}^{5}\left(\delta^{7} 1_{\tau^{\prime}}\right)\left(z_{i}\right)\right) d z_{1} \cdots d z_{5} \\
& \lesssim R^{\epsilon} \lambda^{-1 / 2} \delta^{3} .
\end{aligned}
$$

Hence,

$$
g_{\tau, \lambda}^{2} \lesssim R^{\epsilon} \delta^{10} \lambda^{-1 / 2} \int 1_{\tau^{\prime}}(x-y) d \mu_{\tau}(d y)
$$

Thus proceeding exactly as in the 6 -linear case we have

$$
\left\|\max _{E_{\delta}}\left(\sum_{\tau \in E_{\delta}}\left(\phi_{\tau, \mu} g_{\tau, \lambda}\right)^{2}\right)^{1 / 2}\right\|_{L^{18 / 7}\left(B_{R}\right)} \lesssim R^{\epsilon} \mu \delta^{1 / 9} \lambda^{-1 / 4} \lesssim R^{\epsilon} \mu \delta^{1 / 9} \lambda^{-3 / 4} .
$$


Summarizing, our estimates yield

$$
\begin{aligned}
\left\|\max _{E_{\delta}}\left(\sum_{\tau \in E_{\delta}}\left(\phi_{\tau} g_{\tau}\right)^{2}\right)^{1 / 2}\right\|_{L^{5 / 2}\left(B_{R}\right)} & \lesssim R^{\epsilon} \delta^{-1 / 10} \\
\left\|\max _{E_{\delta}}\left(\sum_{\tau \in E_{\delta}}\left(\phi_{\tau, \mu} g_{\tau, \lambda}\right)^{2}\right)^{1 / 2}\right\|_{L^{18 / 7}\left(B_{R}\right)} & \lesssim R^{\epsilon} \min \left(\mu \lambda^{-3 / 4} \delta^{1 / 9}, \lambda^{1 / 36} \mu^{-1 / 27}\right) \\
& \lesssim R^{\epsilon} \delta^{1 /(9 \cdot 28)} .
\end{aligned}
$$

Then interpolation yields that the improvement is

$$
\frac{5}{1764} \text {. }
$$

\subsection{Estimates on the linear term}

For this term the caps have size $R^{-1 / 2}$ so we can use more direct methods without suffering any significant loss. One such method is using the fact that for a function $f$ supported on $S$ we have, for every $x_{n} \in \mathbb{R}^{n}$,

$$
\|\widehat{f d \sigma}\|_{L^{2}\left(\mathbb{R}^{n-1} \times\left\{x_{n}\right\}\right)} \approx\|f\|_{L^{2}(S)} .
$$

This is referred to as conservation of mass in PDE literature. We briefly give the calculation that leads to this result. Let $x=\left(\bar{x}, x_{n}\right)$. Let $S$ be parametrized by $\xi_{n}=\phi(\bar{\xi})$. Then

$$
\begin{aligned}
\|\widehat{f d \sigma}\|_{L^{2}\left(\mathbb{R}^{n-1} \times\left\{x_{n}\right\}\right)}^{2} & =\int\left|\int f(\bar{\xi}) e^{-i\left(\bar{x} \cdot \bar{\xi}+x_{n} \phi(\bar{\xi})\right)} d \bar{\xi}\right|^{2} d \bar{x} \\
& =\int\left|\int f(\bar{\xi}) e^{x_{n} \phi(\bar{\xi})} e^{-i \bar{x} \cdot \bar{\xi}} d \bar{\xi}\right|^{2} d \bar{x} .
\end{aligned}
$$

Now the inner integral is the Fourier transform of $f(\bar{\xi}) e^{x_{n} \phi(\bar{\xi})}$, thus applying the Plancherel theorem we have

$$
=\int\left|f(\bar{\xi}) e^{x_{n} \phi(\bar{\xi})}\right|^{2} d \bar{\xi}=\int|f(\bar{\xi})|^{2} d \bar{\xi} \approx\|f\|_{L^{2}(S)}^{2} .
$$

To continue the estimation of the linear term we pass from the $c_{\tau}$ to the $b_{\tau}$ variant as before. Thus for the $L^{12 / 5}$ estimate we have

$$
\begin{aligned}
\left\|\left[\sum_{\tau \in E}\left(\phi_{\tau} c_{\tau}\right)^{2}\right]^{1 / 2}\right\|_{L^{12 / 5}\left(B_{R}\right)} & \lesssim\left\|\left[\sum_{\tau \in E}\left(\phi_{\tau} b_{\tau}\right)^{2}\right]^{1 / 2}\right\|_{L^{12 / 5}\left(B_{R}\right)} \\
& \lesssim R^{1 / 8}\left[\sum_{\tau} \int_{B_{R}}\left(\phi_{\tau} b_{\tau}\right)^{12 / 5}\right]^{5 / 12} .
\end{aligned}
$$

Here, on the right-hand side $\tau$ ranges over a partition into $R^{-1 / 2}$ of all of $S$. Due to size of our caps we have $b_{\tau} \lesssim R^{-5 / 2}$. Using this and then conservation of mass we obtain

$$
\lesssim R^{1 / 8+\epsilon}
$$


We estimate in $L^{18 / 7}$ first without using the Kakeya maximal function estimate. First decompose $\phi_{\tau}$ into $\phi_{\tau, \mu}$ exactly as before. Then

$$
\begin{aligned}
\left\|\left[\sum_{\tau \in E}\left(\phi_{\tau, \mu} b_{\tau}\right)^{2}\right]^{1 / 2}\right\|_{L^{18 / 7}\left(B_{R}\right)} & \leq R^{1 / 6}\left(\sum_{\tau}\left\|\phi_{\tau, \mu} b_{\tau}\right\|_{\left.L^{18 / 7\left(B_{R}\right.}\right)}^{18 / 7}\right)^{7 / 18} \\
& \lesssim R^{1 / 6}\left(\mu^{-2 / 21} R^{7 / 2+5 / 2-5 / 2 \cdot 18 / 7+\epsilon}\right)^{7 / 18} \lesssim R^{\epsilon} \mu^{-1 / 27} .
\end{aligned}
$$

Now we shall estimate using Kakeya maximal function bounds. Again we will first pass to probability measures, and then to the maximal function estimate. Thus we write

$$
b_{\tau} \lesssim b_{\tau} * R^{-7 / 2} 1_{\tau^{\prime}} \quad \text { and } \quad\left(b_{\tau}\right)^{2}(x) \lesssim R^{-7 / 2} \int\left(b_{\tau}\right)^{2}(y) 1_{\tau^{\prime}}(x-y) d y,
$$

and estimate using again conservation of mass

$$
\int_{B_{R}}\left(b_{\tau}\right)^{2}(x) d x \lesssim R^{-3 / 2}
$$

Hence

$$
\left(b_{\tau}\right)^{2} \lesssim R^{-5} \int 1_{\tau^{\prime}}(x-y) d \mu_{\tau}
$$

So

$$
\begin{aligned}
& \left\|\left[\sum_{\tau \in E}\left(\phi_{\tau, \mu} b_{\tau}\right)^{2}\right]^{1 / 2}\right\|\left\|_{L^{18 / 7}\left(B_{R}\right)} \leq \mu\right\|\left[\sum_{\tau}\left(b_{\tau}\right)^{2}\right]^{1 / 2} \|_{L^{18 / 7}\left(B_{R}\right)} \\
& \quad \lesssim R^{-(7 / 4+3 / 4)} \mu\left\|\sum_{\tau} 1_{\tau^{\prime}}\left(\cdot-y_{\tau}\right)\right\|_{L^{9 / 7}\left(B_{R}\right)}^{1 / 2} \lesssim R^{-5 / 2+22 / 9+\epsilon} \mu \lesssim R^{-1 / 18+\epsilon} \mu .
\end{aligned}
$$

Interpolation shows that the improvement for this term is, as in the 6-linear case,

$$
\frac{2}{735}
$$

Finally comparing the improvements, we get

$$
\min \left(\frac{2}{735}, \frac{5}{1764}\right)=\frac{2}{735}
$$

and thus we have Theorem 1.

\section{References}

[1] Bennett, J., Carbery A. And Tao T.: On the multilinear restriction and Kakeya conjectures. Acta Math. 196 (2006), no. 2, 261-302.

[2] Bourgain, J.: Besicovitch type maximal operators and applications to Fourier analysis. Geom. Funct. Anal. 1 (1991), no. 2, 147-187. 
[3] Bourgain, J.: Harmonic analysis and combinatorics: How much may they contribute to each other? In Mathematics: Frontiers and perspectives, 13-32. Amer. Math. Soc., Providence, RI, 2000.

[4] Bourgain, J. And Guth, L.: Bounds on oscillatory integral operators based on multilinear estimates. Geom. Funct. Anal. 21 (2011), no. 6, 1239-1295.

[5] Fefferman, C.: Inequalities for strongly singular convolution operators. Acta Math. 124 (1970), no. 1, 9-36.

[6] ŁabA, I.: From harmonic analysis to arithmetic combinatorics. Bull. Amer. Math. Soc. (N.S.) 45 (2008), no. 1, 77-115.

[7] Stein, E. M.: Some problems in harmonic analysis. In Harmonic analysis in Euclidean spaces (Proc. Sympos. Pure Math., Williamstown, Mass., 1978), Part 1, 3-20. Proc. Sympos. Pure Math. 35, Amer. Math. Soc., Providence, RI, 1979.

[8] TAO, T.: A sharp bilinear restriction estimate for paraboloids. Geom. Funct. Anal. 13 (2003), no. 6, 1359-1384.

[9] TAO, T.: Some recent progress on the restriction conjecture. In Fourier analysis and convexity, 217-243. Appl. Numer. Harmon. Anal., Birkhäuser Boston, Boston, MA, 2004.

[10] TaO, T., Vargas, A. And Vega, L.: A bilinear approach to the restriction and Kakeya conjectures. J. Amer. Math. Soc. 11 (1998), no. 4, 967-1000.

[11] TaO, T. And Vargas, A.: A bilinear approach to cone multipliers. I. Restriction estimates. Geom. Funct. Anal. 10 (2000), no. 1, 185-215.

[12] Tomas, P.: A restriction theorem for the Fourier transform. Bull. Amer. Math. Soc. 81 (1975), no. 2, 477-478.

[13] Wolff, T.: An improved bound for Kakeya type maximal functions. Revista Mat. Iberoamericana 11 (1995), no. 3, 651-674.

[14] Wolff, T.: Recent work connected with the Kakeya problem. In Prospects in mathematics (Princeton, NJ, 1996), 129-162. Amer. Math. Soc., Providence, RI, 1999.

Received September 21, 2012.

FAruk Temur: Department of Mathematics, University of Illinois at UrbanaChampaign, Urbana, IL 61801, USA.

E-mail: temur1@illinois.edu 